Case Report

\title{
Localized Pigmented Villonodular Synovitis of the Hip: Sudden-Onset Pain Caused by Torsion of the Tumor Pedicle
}

\author{
Kiyokazu Fukui, ${ }^{1}$ Ayumi Kaneuji, ${ }^{1}$ Eriko Kinoshita, ${ }^{2}$ Yuhei Numata, \\ Takayuki Nojima, ${ }^{2}$ and Tadami Matsumoto ${ }^{1}$ \\ ${ }^{1}$ Department of Orthopaedic Surgery, Kanazawa Medical University, 1-1 Daigaku, Uchinada-machi, Kahokugun, \\ Ishikawa 920-0293, Japan \\ ${ }^{2}$ Department of Pathology and Medical Laboratory, Kanazawa Medical University, Daigaku, Uchinada-machi, Kahokugun, \\ Ishikawa 920-0293, Japan
}

Correspondence should be addressed to Kiyokazu Fukui; 66406kf@kanazawa-med.ac.jp

Received 5 August 2013; Accepted 1 October 2013

Academic Editors: I.-H. Choi and T. Yasuda

Copyright (c) 2013 Kiyokazu Fukui et al. This is an open access article distributed under the Creative Commons Attribution License, which permits unrestricted use, distribution, and reproduction in any medium, provided the original work is properly cited.

\begin{abstract}
Pigmented villonodular synovitis is a rare, benign, but potentially locally aggressive disease that should be considered in younger patients who present with monoarticular joint symptoms and pathology. We present the case of a 33-year-old woman with a mass arising from her right hip joint that was examined using a multimodal radiological approach. Because her clinical presentation mimicked that of synovial osteochondromatosis of the hip, surgical dislocation was performed. Histopathological examination of the resected specimen confirmed the diagnosis of localized pigmented villonodular synovitis, with the mass consisting of proliferation of fibrohistiocytic cells, abundant hemosiderin, foamy histiocytes, and occasional giant cells. Because of the presence of tumor necrosis, we hypothesize that torsion of the tumor pedicle was the cause of acute presentation.
\end{abstract}

\section{Introduction}

The term pigmented villonodular synovitis (PVNS) was coined by Jaffe et al. in 1941 to describe a group of localized or diffuse synovium-based lesions involving tendon sheaths, less commonly joints, and rarely bursae [1]. PVNS is a benign proliferative disorder of the synovium of unknown origin [2-10]. Localized versus diffuse forms of PVNS may cause different clinical symptoms. It typically appears as an intra-articular effusion of low intensity on both $\mathrm{T}_{1}$ and $\mathrm{T}_{2}$-weighted images because of hemosiderin deposits, with thick fibrous tissue, synovial hyperplasia, bone erosion, and preserved bone density and joint-space width [3-5]. PVNS can be demonstrated as a dark lesion on all pulse sequences of magnetic resonance imaging (MRI) because of the ferromagnetic properties of hemosiderin [11]. PVNS of the hip is a relatively uncommon disease. At any body site, PVNS has an estimated worldwide incidence of 1.8 per million cases per year; a hip is involved in $15 \%$ of those cases $[12,13]$. Here we describe the case of 33-year-old woman who presented with sudden-onset hip pain and an intra-articular mass in the left hip joint. A diagnosis of PVNS should be kept in mind in younger patients who present with monoarticular arthritis, especially when it is associated with bony erosions or a soft-tissue component.

\section{Case Report}

The patient was a 33-year-old woman who had had a mildly limited range of motion in her left hip for a long time. In October 2012, she experienced sudden, severe pain in her left hip without any antecedent trauma or episode. The pain affected her not only when she moved the hip but also when she was at rest. Her condition had been diagnosed at another hospital as synovial osteochondromatosis of the hip, for which she was given an anti-inflammatory drug (loxoprofen, $60 \mathrm{mg}$, three times daily for 10 days). Although the severe pain disappeared about 10 days after onset and only vague discomfort and pain in a specific posture had 


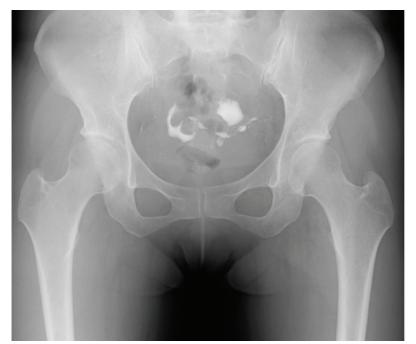

(a)

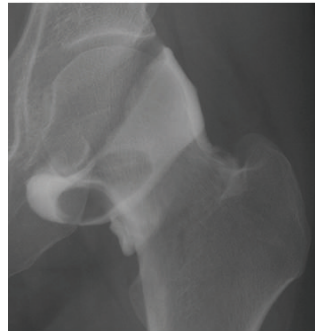

(b)

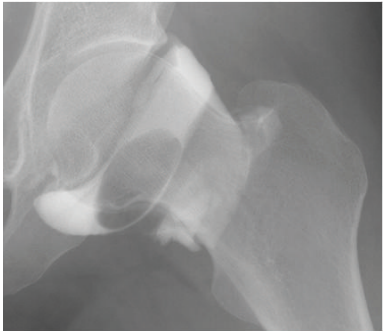

(c)

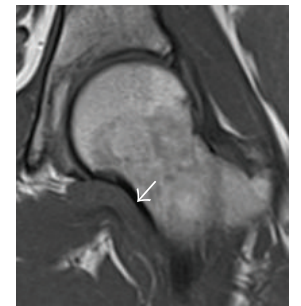

(d)

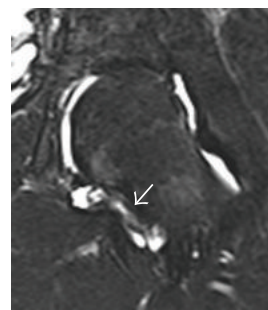

(e)

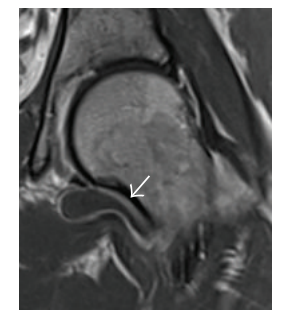

(f)

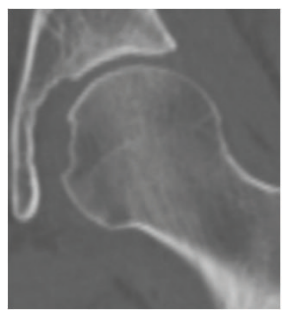

(g)

FIGURE 1: (a) An initial anterior radiograph shows no significant findings. (b, c) Enhanced stress radiographs reveal a mobile intra-articular mass. (d-f) Magnetic resonance images: $(d)$ a coronal $\mathrm{T}_{1}$-weighted image shows a low-intensity mass lesion located inferior to the hip joint; (e) a coronal short $\tau$ inversion recovery sequence shows a mass with both low- and high-intensity areas; (f) a coronal enhanced $\mathrm{T}_{1}$-weighted image shows marginal enhancement of the mass. (g) Computed tomography image shows erosive changes on the fossa acetabuli and enthesis of the ligamentum teres of the femur.

remained, she was referred to our hospital for a surgical treatment at 3 weeks after pain onset. The range of motion in the affected hip was $130^{\circ}$ in flexion, $15^{\circ}$ in extension, $30^{\circ}$ in abduction, $20^{\circ}$ in adduction, $45^{\circ}$ in external rotation, and $15^{\circ}$ in internal rotation. Findings on the Patrick test were positive, the anterior impingement sign was present, and she had mild tenderness of the Scarpa triangle. Preoperative blood tests revealed no evidence of diabetes, rheumatoid arthritis, infection, or abnormality in renal or liver function. Although initial radiographs, obtained 1 month after pain onset, revealed no significant findings, magnetic resonance images showed marginal enhancement of a mass located inferior to the hip joint (Figure 1). We performed surgical dislocation of the joint using the technique described by Ganz et al. [14] for tumor excision, with the patient receiving general anesthesia. We excised a whitish-yellow encapsulated tumor, $4 \times 2 \times 1 \mathrm{~cm}^{3}$, arising from the anteromedial synovium (Figure 2). We assumed that the pain was not caused by the tumor putting pressure on the surrounding area because the tumor was a soft elastic mass and could move easily. The mobility of the tumor was seen preoperatively in the enhanced stress radiographs (Figure 1). Synovectomy in the fossa acetabuli was also performed. Microscopy revealed cells of mononuclear stromal origin with hyalinization and multinucleated giant cells. There was hemosiderin pigment in macrophages and in the extracellular space. The nuclei of the mononuclear stromal cells and multinucleated giant cells had disappeared because of tumor necrosis (Figure 3).

\section{Discussion}

PVNS is a rare, benign, proliferative condition of the synovium involving a joint, bursa, or tendon sheath. Its etiology

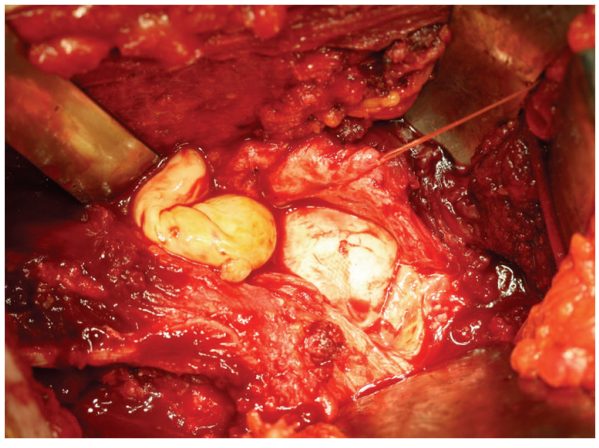

FIgURE 2: Excision of the synovial tumor using the Ganz surgical dislocation approach.

is unknown, although a neoplastic or inflammatory origin has been suggested $[6,15]$. It affects patients in the age group of 20 to 40 years. Prevalence by sex has been reported to range between a $2: 1$ male-to-female ratio and a slight female preponderance [16]. Knee involvement accounts for $80 \%$ of cases, followed by the hip, ankle, shoulder, and elbow [17]. Localized PVNS is a relatively rare form, representing $27 \%$ of PVNS cases [5]. In the hip, the disease is usually of the diffuse villonodular type [18]. The average time lapse between symptom onset and diagnosis is 2 years. However, there are outliers in whom the disease was diagnosed as soon as a few days after symptom onset to as long as 12 years afterward. Localized PVNS is commonly seen in the third decade of life $[3,4]$, with mononuclear stromal cell hyperplasia, multinuclear giant cells, and macrophages with hemosiderin [3]. Hemosiderin can accumulate in both macrophages and extracellular compartments; its accumulation is responsible 


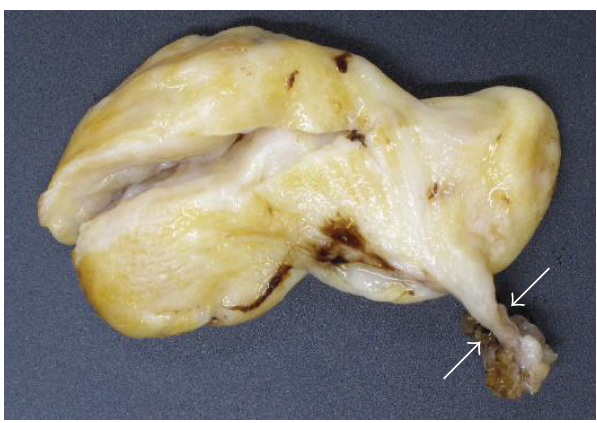

(a)

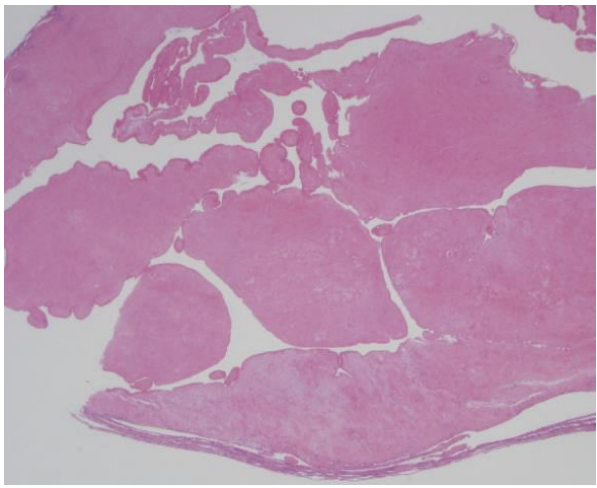

(c)

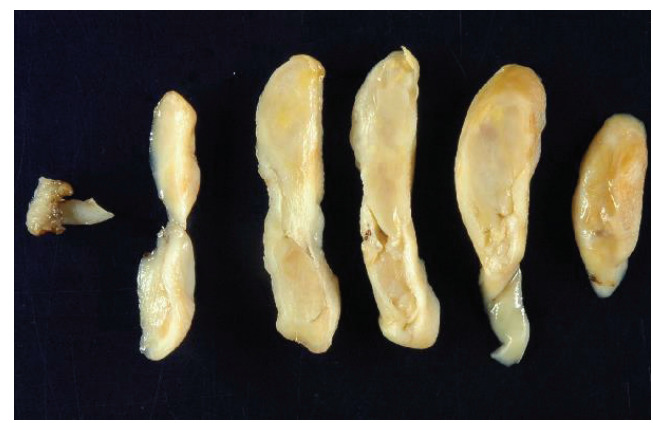

(b)

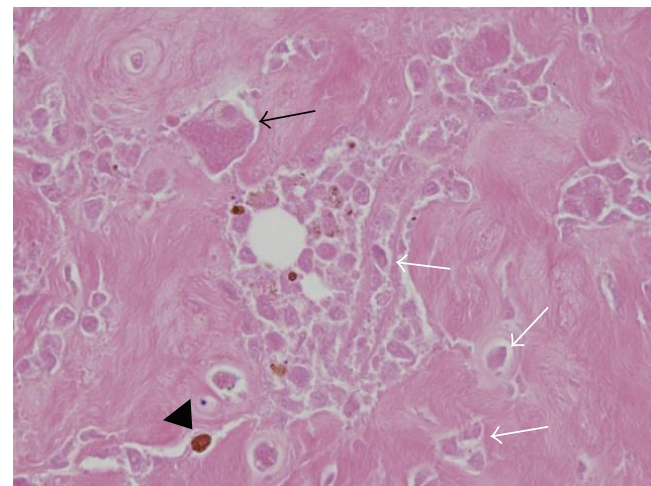

(d)

Figure 3: (a) The excised tumor had a pedicle (white arrows), and (b) the cut sections revealed tumor necrosis except in the margins. (c) A histological image shows a nodular and papillary structure and the presence of synovial cells, histiocytes, and hyperplasia (original magnification $\times 1.25$; hematoxylin and eosin staining). (d) Histopathological findings: enucleated mononuclear stromal cells (white arrows) and multinuclear giant cells (black arrow), with hemosiderin in the cytoplasm (black arrowhead) (original magnification $\times 100$; hematoxylin and eosin staining).

for the "pigmented" portion of the disease's name. Hemorrhage and inflammatory infiltration can take place in the tumor. The key features of PVNS seen on plain radiographs include dense soft-tissue swelling, scalloped bony erosions with sclerotic margins, and joint-space preservation during the early stage of the disease. The absence of juxta-articular osteopenia and osteophytes is characteristic [19]. Generally, sudden-onset torsion of an ovarian tumor pedicle causes severe pain in the lower abdomen [20, 21] and stagnation of the tumor's venous return, which leads to inflammation and necrosis of the tumor. In our patient, we were able to document such necrosis, and we speculate that it was responsible for the sudden onset of symptoms caused by the same mechanism of torsion as in ovarian tumor pedicles. Whether through arthroscopy or arthrotomy, excision is sufficient for localized PVNS, but if the tumor is diffuse, total synovectomy is necessary $[2-5,10]$. In this case, there was no need for a biopsy to determine if it was a diffuse PVNS because we had already diagnosed it as either a localized PVNS or a synovial osteochondromatosis. Radiotherapy for PVNS is controversial [3]. We performed a Ganz surgical dislocation because it was necessary not only to excise the tumor but also to perform a synovectomy in the fossa acetabuli. In summary, sudden severe pain in the hip joint may be found, during surgery, to be caused by PVNS, a diagnosis confirmed after surgical removal of a synovial tumor. Sudden pain onset is an unusual clinical presentation for localized PVNS, so surgeons should keep in mind that it is possible that torsion of a tumor pedicle plays a role in the abruptness of symptom onset.

\section{Disclosure}

The authors of this paper required no funding for the creation of this study. Additionally, the authors have no financial disclosures to report. This paper has not been submitted to any other journal, and its text is original.

\section{Acknowledgments}

Medical editor Katharine O’Moore-Klopf, ELS (East Setauket, NY, USA) provided professional English-language, editing of this paper. Each author certifies that he has no commercial associations (e.g., consultancies, stock ownership, equity interest, or patent/licensing arrangements) that might pose a conflict of interests in connection with the submitted paper. Each author certifies that his institution approved the reporting of this case and that all investigations were conducted in conformity with ethical principles of research. 


\section{References}

[1] H. L. Jaffe, L. Lichtenstein, and C. J. Sutro, "Pigmented villonodular synovitis, bursitis and tenosynovitis," Archives of Pathology \& Laboratory Medicine, vol. 31, pp. 731-765, 1941.

[2] S. W. Weiss and J. R. Goldblum, "Enzinger and Weiss's soft tissue tumors," in Benign Tumors and Tumor-Like Lesions of Synovial Tissue, F. Enzinger and S. Weiss, Eds., pp. 1037-1062, Mosby, Philadelphia, Pa, USA, 4th edition, 2001.

[3] M. Szendroi and A. Deodhar, "Synovial neoformations and tumours," Bailliere's Best Practice and Research in Clinical Rheumatology, vol. 14, no. 2, pp. 363-383, 2000.

[4] H. S. Schwartz, K. K. Unni, and D. J. Pritchard, "Pigmented villonodular synovitis. A retrospective review of affected large joints," Clinical Orthopaedics and Related Research, no. 247, pp. 243-255, 1989.

[5] E. de Visser, R. P. H. Veth, M. Pruszczynski, T. Wobbes, and L. B. A. van de Putte, "Diffuse and localized pigmented villonodular synovitis: evaluation of treatment of 38 patients," Archives of Orthopaedic and Trauma Surgery, vol. 119, no. 7-8, pp. 401-404, 1999.

[6] S. Oehler, H. G. Fassbender, D. Neureiter, C. Meyer-Scholten, T. Kirchner, and T. Aigner, "Cell populations involved in pigmented villonodular synovitis of the knee," Journal of Rheumatology, vol. 27, no. 2, pp. 463-470, 2000.

[7] R. C. Martin, D. L. Osborne, M. J. Edwards, W. Wrightson, and K. M. McMaster, "Giant cell tumor of tendon sheath, tenosynovial giant cell tumor, and pigmented villonodular synovitis: defining the presentation, surgical therapy and recurrence," Oncology Reports, vol. 7, no. 2, pp. 413-419, 2000.

[8] A. B. Goldman and E. F. DiCarlo, "Pigmented villonodular synovitis. Diagnosis and differential diagnosis," Radiologic Clinics of North America, vol. 26, no. 6, pp. 1327-1347, 1988.

[9] A. S. Rao and V. J. Vigorita, "Pigmented villonodular synovitis (giant-cell tumor of the tendon sheath and synovial membrane). A review of eighty-one cases," Journal of Bone and Joint Surgery, vol. 66, no. 1, pp. 76-94, 1984.

[10] M. Aşik, L. Erlap, L. Altinel, and O. Cetik, "Localized pigmented villonodular synovitis of the knee," Arthroscopy, vol. 17, no. 6, p. E23, 2001.

[11] T. H. Hughes, D. J. Sartoris, M. E. Schweitzer, and D. L. Resnick, "Pigmented villonodular synovitis: MRI characteristics," Skeletal Radiology, vol. 24, no. 1, pp. 7-12, 1995.

[12] B. W. Myers and A. T. Masi, "Pigmented villonodular synovitis and tenosynovitis: a clinical epidemiologic study of 166 cases and literature review," Medicine, vol. 59, no. 3, pp. 223-238, 1980.

[13] L. Vastel, P. Lambert, G. de Pinieux, O. Charrois, M. Kerboull, and J.-P. Courpied, "Surgical treatment of pigmented villonodular synovitis of the hip," The Journal of Bone and Joint Surgery. American, vol. 87, no. 5, pp. 1019-1024, 2005.

[14] R. Ganz, T. J. Gill, E. Gautier, K. Ganz, N. Krügel, and U. Berlemann, "Surgical dislocation of the adult hip a technique with full access to the femoral head and acetabulum without the risk of avascular necrosis," The Journal of Bone and Joint Surgery. British, vol. 83, no. 8, pp. 1119-1124, 2001.

[15] P. F. Choong, H. Willen, M. Nilbert et al., "Pigmented villonodular synovitis. Monoclonality and meteastasis-a case for neoplastic origin?" Acta Orthopaedica Scandinavica, vol. 66, no. 1, pp. 64-68, 1995.

[16] K. M. Kallas, L. Vaughan, P. Haghighi, and D. Resnick, "Pigmented villonodular synovitis of the hip presenting as a retroperitoneal mass," Skeletal Radiology, vol. 30, no. 8, pp. 469474, 2001.

[17] S. Gitelis, D. Heligman, and T. Morton, "The treatment of pigmented villonodular synovitis of the hip. A case report and literature review," Clinical Orthopaedics and Related Research, no. 239, pp. 154-160, 1989.

[18] P. Aglietti, G. V. di Muria, E. A. Salvate, and G. Stringa, "Pigmented villonodular synovitis of the hip joint (review of the literature and report of personal case material)," Italian Journal of Orthopaedics and Traumatology, vol. 9, no. 4, pp. 487-496, 1983.

[19] J. A. Narváez, J. Narváez, C. Aguilera, E. De Lama, and F. Portabella, "MR imaging of synovial tumors and tumor-like lesions," European Radiology, vol. 11, no. 12, pp. 2549-2560, 2001.

[20] H. C. Chang, S. Bhatt, and V. S. Dogra, "Pearls and pitfalls in diagnosis of ovarian torsion," Radiographics, vol. 28, no. 5, pp. 1355-1368, 2008.

[21] E. J. Lee, H. C. Kwon, H. J. Joo, J. H. Suh, and A. C. Fleischer, "Diagnosis of ovarian torsion with color Doppler sonography: depiction of twisted vascular pedicle," Journal of Ultrasound in Medicine, vol. 17, no. 2, pp. 83-89, 1998. 


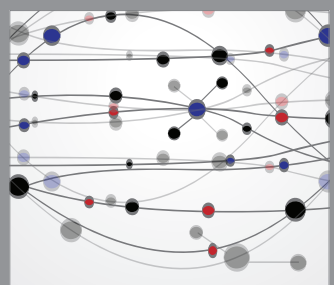

The Scientific World Journal
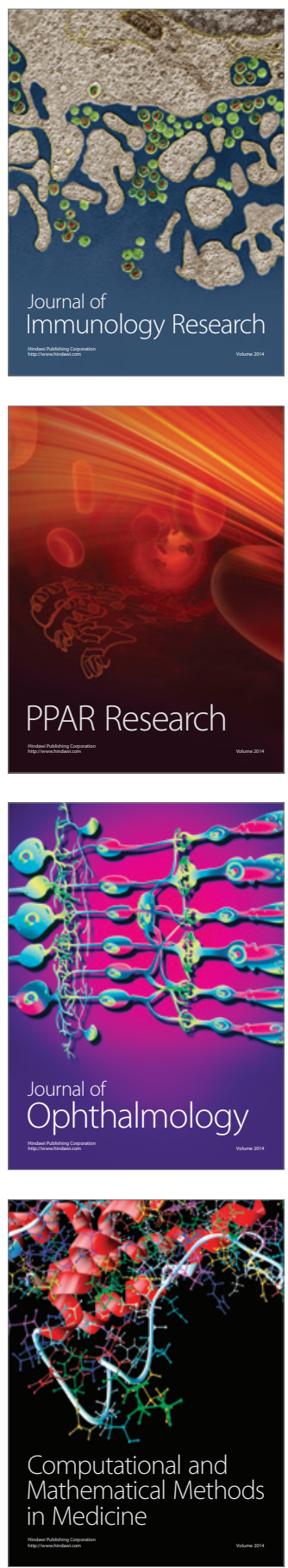

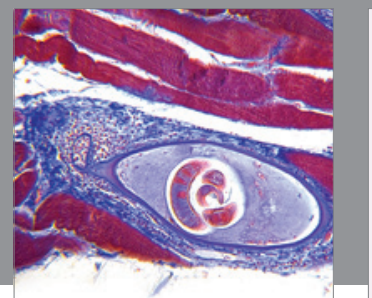

Gastroenterology

Research and Practice
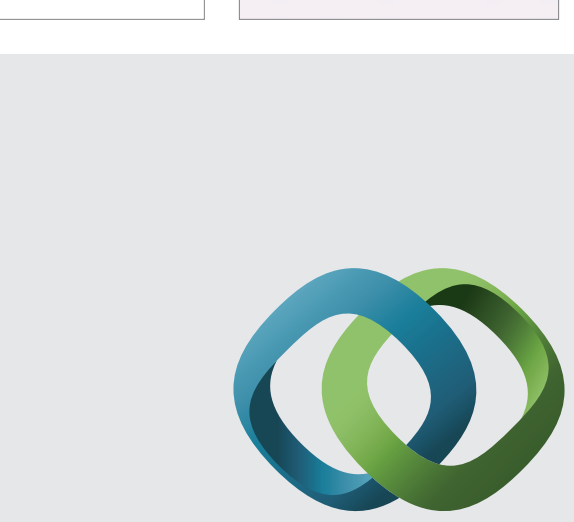

\section{Hindawi}

Submit your manuscripts at

http://www.hindawi.com
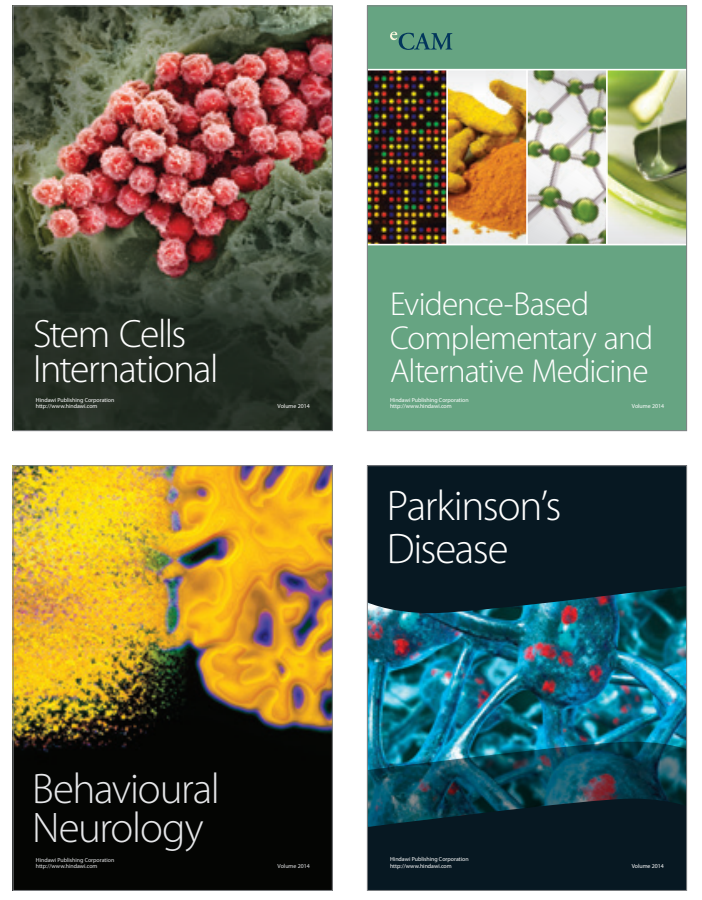
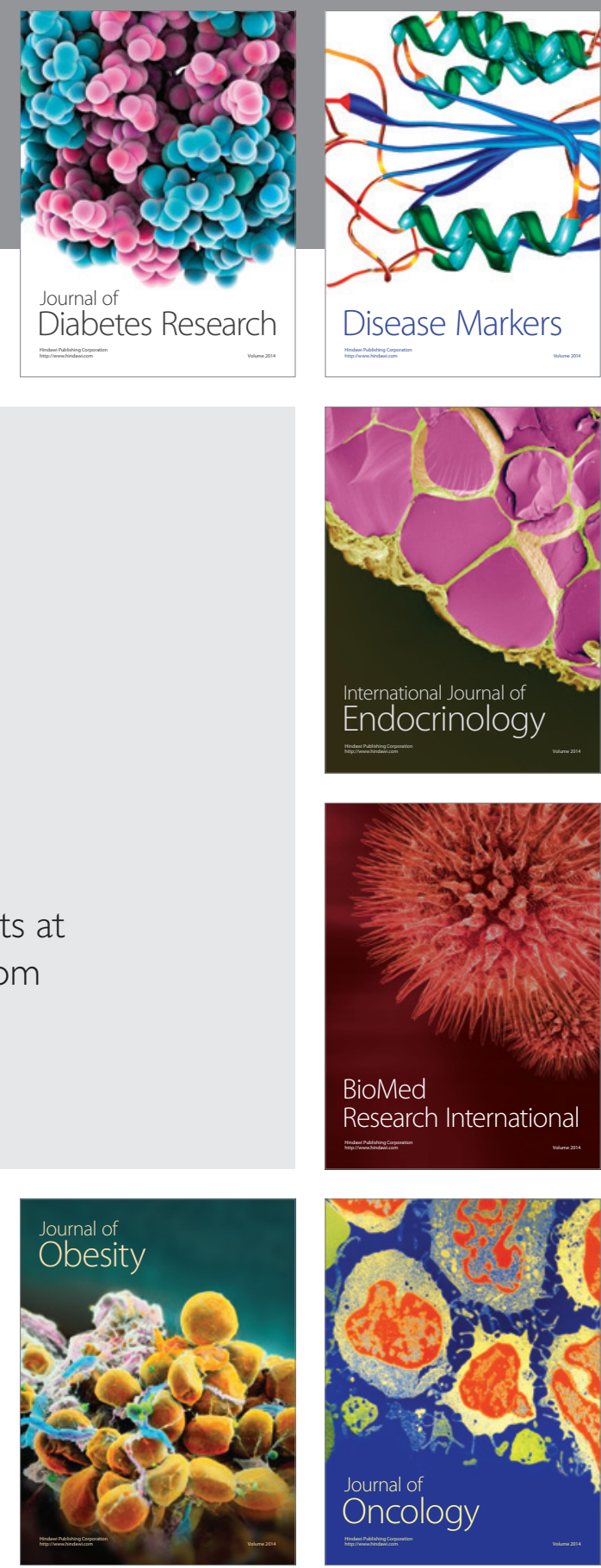

Disease Markers
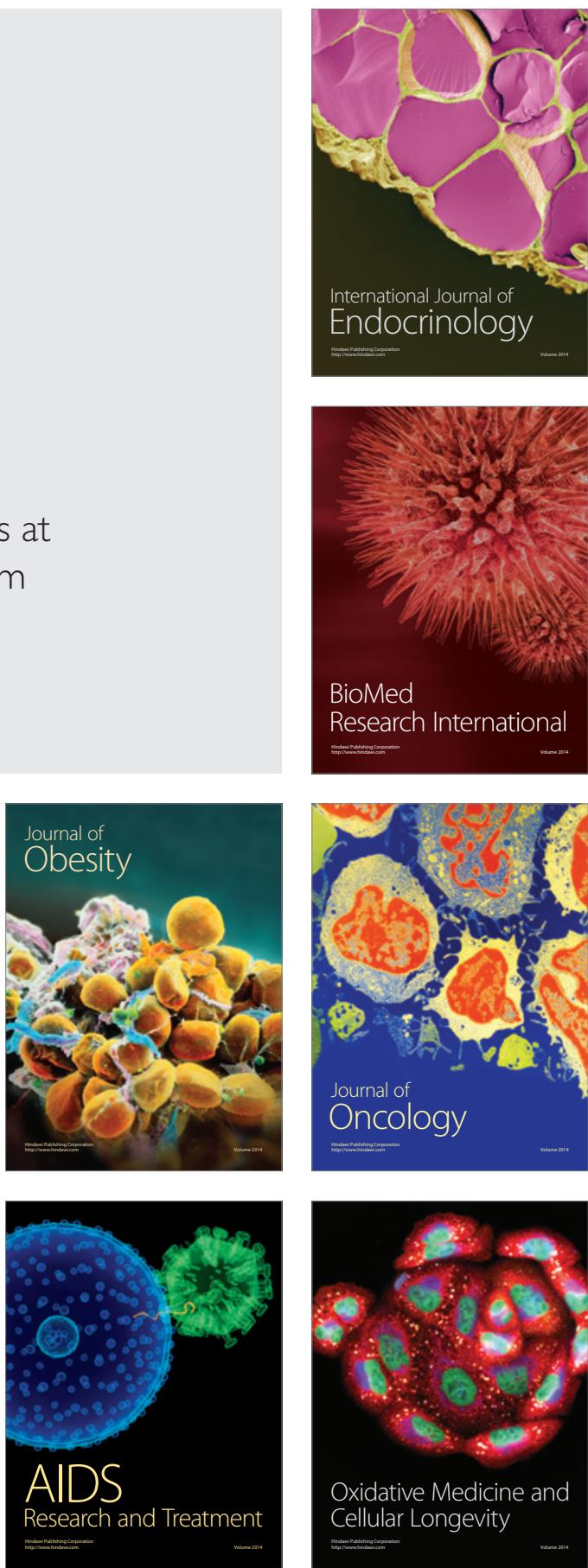